\title{
Born to run... ma anche per camminare. Attività motoria, il primo pilastro dell' evoluzione umana
}

Giornale di Tecniche Nefrologiche e Dialitiche 2019, Vol. 31(2) 142-145

(C) The Author(s) 2019

Article reuse guidelines:

sagepub.com/journals-permissions

DOI: $10.1177 / 0394936219851937$

journals.sagepub.com/home/gtn

(S)AGE

\author{
Alessandro Capitanini' e David Mariani²
}

\begin{abstract}
Born to run ... but also to walk. Motor activity: the first pillar of human evolution.

Our evolution has been based on the ability of moving in order to find food, escape or fight. Afterwards, the first social organizations reduced the risk of aggressions by predators by allowing a shared hunting activity and collection of foods determining, this way, less energy required lifestyle. Thanks to the subsequent progresses in human society, we have therefore moved from hunting and agriculture to industrialization. For about seventy years, an apparently unstoppable progression towards a sedentary lifestyle has been going on, with a gradual loss of some of our body's functions, up to the so-called "disuse syndrome". The change can also be observed in the type of prevalent pathologies: from acute/infectious/transmissible/epidemic, to chronic/degenerative/dysmetabolic and, unfortunately, increasingly pandemic. Therefore, physical activity can be both a preventive and therapeutic solution.
\end{abstract}

\section{Keywords}

Evolution, Physical exercise, Lifestyle, Sedentariety, Chronic diseases, Endurance activity, Strenght exercises, Prevention.

\section{Introduzione}

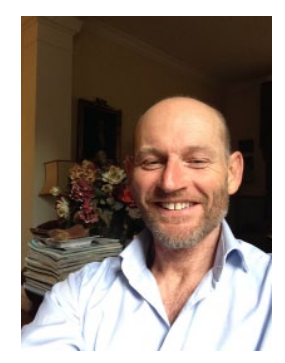

La nostra storia evolutiva si è basata sulla capacità di muoversi, per la ricerca di cibo e per fuggire o combattere di fronte ad aggressioni di propri simili o animali feroci. L'ambiente in cui l'Homo sapiens si è evoluto necessitava di una notevole capacità fisica per sopravvivere, tanto che il sistema cardiovascolare umano si è adattato e sviluppato per rispondere alle sue richieste. ${ }^{1}$ Ma la capacità di muoversi, allora come oggi, può essere una questione di vita o di morte per molti animali terrestri incluso l'uomo, anche se non proprio come lo è stato nelle sue prime fasi evolutive. ${ }^{2}$

L'evoluzione della società umana è caratterizzata da una correlazione inversa tra la spesa energetica da parte dei sistemi cardiovascolare e muscolare. ${ }^{3}$ L'essere umano da solo è inadatto a sopravvivere in un ambiente selvaggio come quello primordiale ed in parte, pur se per motivi diversi, anche in quello attuale. Da questo punto di vista è stata fondamentale la socializzazione (tribale) tra i primi essere umani, come poi in tutte le restanti età dell'uomo, che ha permesso loro di risparmiarsi molte fatiche ovviando una gran parte del fardello fisico dovuto anche solo alla sopravvivenza.

Le prime organizzazioni sociali infatti hanno consentito di ridurre i rischi dovuti ai predatori e di condividere la caccia e la raccolta di cibo. Dal 7000 a.C. lo sviluppo dell'agricoltura ha facilitato 1'abbandono dalla ricerca itinerante, costante e difficoltosa del cibo permettendo così uno stile di vita energeticamente meno dispendioso. Questo periodo, noto come "Rivoluzione Neolitica", ha prodotto l'ascesa dell'Homo sapiens al vertice della catena alimentare e gli ha permesso di ottenere una drastica riduzione dei costi

\footnotetext{
'Nefrologia e Dialisi Ospedale di Pistoia, Azienda Sanitaria Toscana Centro, Italy

2Healthy Habits Academy Director, Italy

Corrispondenza:

David Mariani, Healthy Habits Academy Director, Italy.

E-mail: d.mariani@healthyhabits.it
} 
energetici dovuti alla sopravvivenza. Ciononostante l'uomo ha dovuto continuare a muoversi e lavorare duramente per sopravvivere. Caccia e agricoltura hanno comunque rappresentato sempre una fonte di spesa energetica non indifferente e tale da mettere in moto quasi costantemente il sistema muscolo-scheletrico e quello cardiovascolare tanto da fargli prevenire molte di quelle malattie croniche che oggi colpiscono l'uomo moderno. Per la maggior parte della storia umana, l'attività fisica necessaria per la sopravvivenza è stata più che sufficiente per sostenere un discreto stato di salute, al punto tale che -a differenza della situazione attualela maggior parte degli individui moriva per malattie infettive piuttosto che per malattie dismetaboliche croniche.

Con il progresso della società umana, si è passati dalla caccia all'agricoltura e da questa all'industrializzazione: lo sforzo fisico obbligato dall' ambiente è così progressivamente diminuito così come sono diminuite le richieste ai sistemi cardiovascolare e muscoloscheletrico. Tuttavia, dalla seconda metà del $20^{\circ}$ secolo è avvenuto un cambiamento drastico di qualità, quantità e soprattutto velocità. I discendenti dell'Homo sapiens hanno saputo 'plasmare' a proprio 'comodo' gli ambienti naturali e sociali in modo tale da permettere di ridurre sempre più le necessità di faticare e perfino di muoversi. Tutto ciò grazie ad attività lavorative basate sempre più sulla conoscenza e sempre meno sull'uso del corpo umano, della fatica, del movimento, insomma sull'uso dell'apparato muscoloscheletrico e tendineo e, di conseguenza, di quello cardiovascolare.

L'uomo moderno vive in un ambiente che ha esplicitamente progettato e disegnato per eliminare il lavoro fisico quanto più possibile. All'inizio del XX secolo il contributo della spesa energetica umana nelle attività lavorative era di circa il $30 \%$; oggi quella cifra è inferiore all' $1 \% .{ }^{4-6}$ Da circa settanta anni è iniziato un cambiamento senza precedenti del modo di vivere della specie umana, caratterizzato da una progressione apparentemente inarrestabile verso la sedentarietà, con progressiva perdita di alcune funzionalità corporee sino ad arrivare alla cosiddetta "disuse syndrome". 7 Possiamo affermare senza timore di smentita che, in modo concomitante e consequenziale, si è assistito anche ad una transizione epidemiologica delle patologie umane passate dalle malattie prevalentemente acute, infettive e trasmissibili, financo in forma epidemica, a quelle dismetabolico-degenerative prevalentemente croniche ed in forma anche pandemica. ${ }^{8-10}$ Come afferma Blair nel suo studio "Aerobics Center Longitudinal Study", ${ }^{11}$ le morti evitabili percentualmente più sostanziose sono quelle dovute a low fitness cardiovascolare.

Infatti, nelle persone affette dalle patologie croniche di cui sopra, che oggi in Italia rappresentano il $40 \%$ dell' intera popolazione, il mantenimento o la ripresa di una corretta dose di attivitá fisica può rappresentare un'ottima opportunità non solo per prevenire la malattia stessa ma anche per limitarne la progressione, producendo un miglioramento significativo della qualità della vita ${ }^{11}$ ed un notevole risparmio da parte del sistema sanitario. ${ }^{12}$ Sempre più associazioni, quali ad esempio l'American Hearth Association, ${ }^{13}$ dichiarano che lo stile di vita sedentario rappresenta il più importante fattore di rischio modificabile per la patologia cardiovascolare. E sulla stessa onda è sempre più orientata anche la letteratura scientifica: si pensa ad esempio che la pratica dell'esercizio fisico in età giovanile è fondamentale per evitare l'insorgenza di numerose patologie neurodegenerative in età adulta. ${ }^{14}$ Inoltre, una messe di dati in favore dell'attività fisica come prevenzione o terapia consociata delle più diffuse patologie metaboliche, come diabete, obesità ed ipertensione arteriosa, si sta raccogliendo sempre nella letteratura scientifica internazionale. (13-14).

Ovviamente, come tutti (o quasi) i farmaci anche l'esercizio fisico necessita di giuste dosi e di alcune attenzioni: è chiaro però è chiaro e deve essere ribadito che non è l'attività fisica ad essere pericolosa ma la sua dose, che deve essere ovviamente adattata alle condizioni cliniche del soggetto. ${ }^{15}$ In estrema sintesi, l'esercizio fisico o attivitá fisica può essere diviso in due grandi comparti, uno relativo al potenziamento della forza muscolare (ad esempio potenziamento con pesi o elastici a corpo libero), e uno relativo al potenziamento della funzionalità cardiovascolare (ad esempio andare in bici, nuotare, camminare velocemente, correre, ballare, ecc.). Questi due comparti esercitano diversamente la loro funzione preventiva.

Le attività aerobiche o di endurance, ad esempio, hanno dimostrato di essere efficacissime nella prevenzione e nella cura di quasi tutte le patologie croniche come ictus, infarti, ipertensione, diversi tipi di tumori, osteoporosi, depressione, malattie del metabolismo, obesità, artrosi, artrite, malattie degenerative come Parkinson e Alzheimer., ${ }^{4,9,10,16}$

Le attività di questo tipo si caratterizzano per una maggiore durata ma una minore intensità dello sforzo. Viceversa, le attività di potenziamento o anaerobiche, caratterizzate da elevati livelli di forza ma tempi di esercizio inferiori e con pause, si rivelano invece efficaci nella prevenzione dell'osteoporosi, del diabete e anche dell'ipertensione arteriosa, contrariamente a quanto sostenuto in un primo momento dalla comunità scientifica. ${ }^{17}$ Gli esercizi di forza permettono -ove correttamente eseguitidi migliorare e soprattutto mantenere autonomia e autosufficienza nell'anziano, contrastando la perdita di massa ossea e muscolare tipiche della vecchiaia, inviando un chiaro messaggio di ringiovanimento alle cellule per mezzo della produzione di citochine infiammatorie. ${ }^{18} \mathrm{Gli}$ esercizi di forza e quelli aerobici possono anche aiutare il mantenimento di una vita sessuale attiva in età anche avanzate, favorendo il mantenimento della funzionalità circolatoria locale e generale e degli ormoni preposti. ${ }^{19}$

L'elemento chiave per le persone "malate" di sedentarietà o affette da patologie, e che decidono di riattivarsi, consiste nell'individuare, dopo un'appropriata e sempre consigliata valutazione medica, un livello di partenza adeguato e un'attività che risulti gradita. Infatti, il maggior rischio per i neo-riattivati é rappresentato proprio dal dolore e dalla fatica eccessivi, che sono assicurati quando si eccede nel 


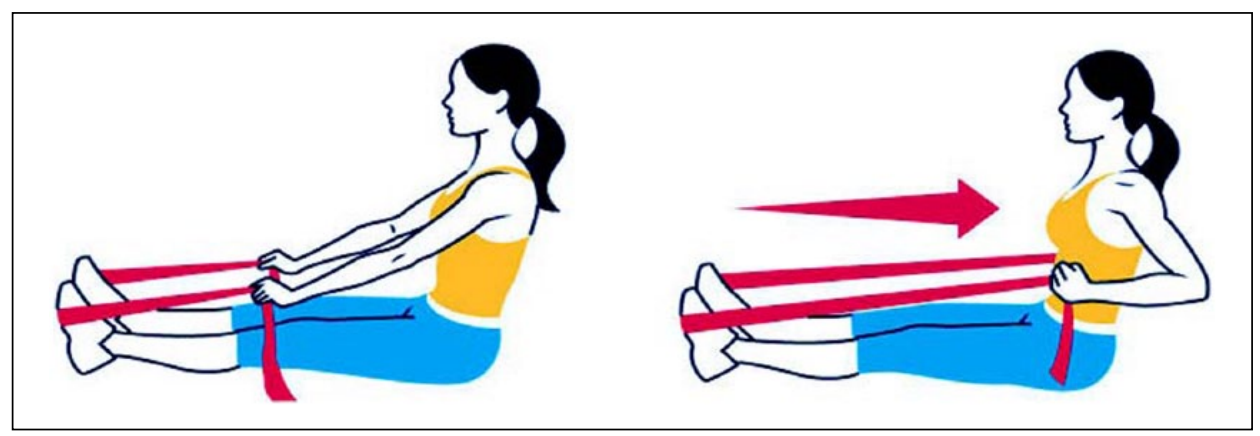

Figura I.

dosaggio dell'attività stessa: tali sensazioni negative sono un potente dissuasore nel proseguimento dell'attività, per cui sono da evitare. Da qui l'importanza di stabilire un livello di partenza adeguato in quanto modo migliore per evitare questo effetto collaterale con attività di blocco. Un altro utile suggerimento derivante dall'esperienza é la continuità: cercare di praticare a piccole dosi ma quotidianamente, o quasi, l'attività motoria si confà perfettamente con l'evitare l'eccessivo dosaggio. Un errore nella prima fase, infatti, potrebbe portare all'abbandono della nuova attività e al consolidarsi dell'errata convinzione del "non ci riuscirò mai!".

Da tenere presente che "poco é comunque molto più di niente". A titolo di esempio, per un sedentario cronico, anche 10 minuti di cammino rappresentano già un livello sufficiente di partenza, in grado di provocare adattamenti iniziali, positivi e significativi. Il progressivo e soprattutto sostenibile incremento della dose, permetterà poi di non innescare mai il dolore né la troppa fatica. Al contrario, la sensazione di "facilità" farà percepire alla persona una sensazione di adeguatezza e motivazione a continuare a fare la nuova attività: la motivazione é la molla più potente per la prosecuzione o meno di una qualsiasi attività.

Si consideri che il conteggio degli atti respiratori in un minuto potrebbe essere un semplicissimo strumento economico ed alla portata di tutti per monitorare l' adeguatezza della nostra attività (quasi come un cardio-frequenzimetro).

Per essere certi che stiamo facendo un lavoro adeguato dovremmo arrivare a fare da 20 a 24 atti respiratori al minuto (salvo determinate condizioni patologiche). Tale attività corrisponde ad una via intermedia tra l'attività respiratoria a riposo (12-18 $\mathrm{rsp} / \mathrm{min})$ e quella affannosa dovuta ad uno sforzo massimale (30-35 rsp/min). Nel caso sia disponibile il cardio-frequenzimetro, vale la vecchia formula 220 - (etá * $65 \%-70 \%$ ). ${ }^{20}$

Negli esercizi di forza possiamo invece iniziare con un semplice elastico ed eseguire un paio di esercizi, come il movimento del vogatore (figura 1), e delle accosciate semplici, come alzarsi da una sedia non molto alta per 10-12 volte (figura 2), e con dei push-ups facilitati (figura 3) 21, 22 $^{2}$ due o tre volte a settimana a giorni alterni.

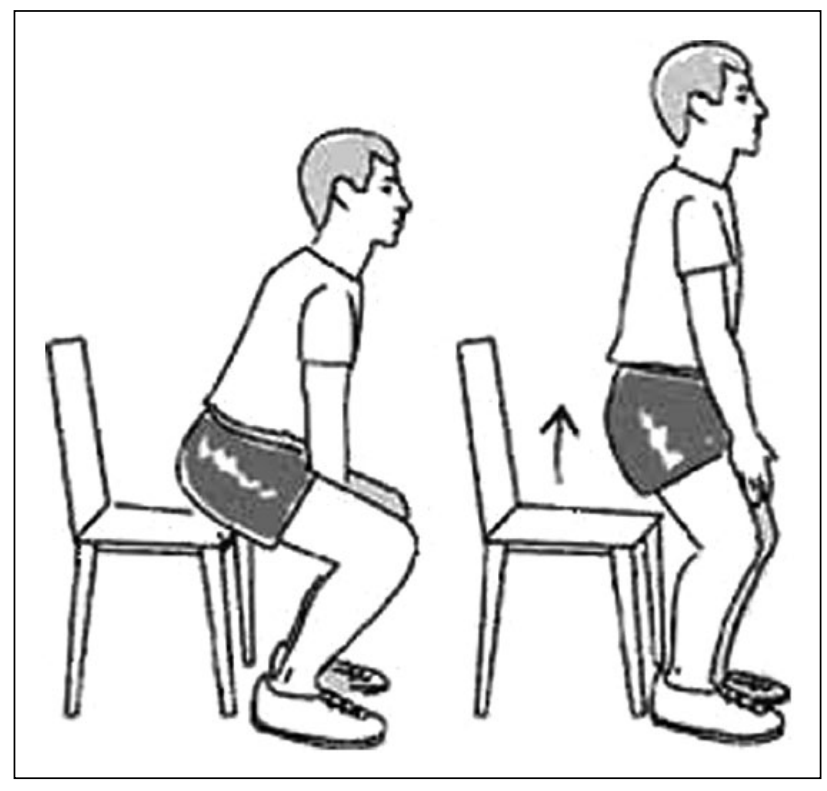

Figura 2.

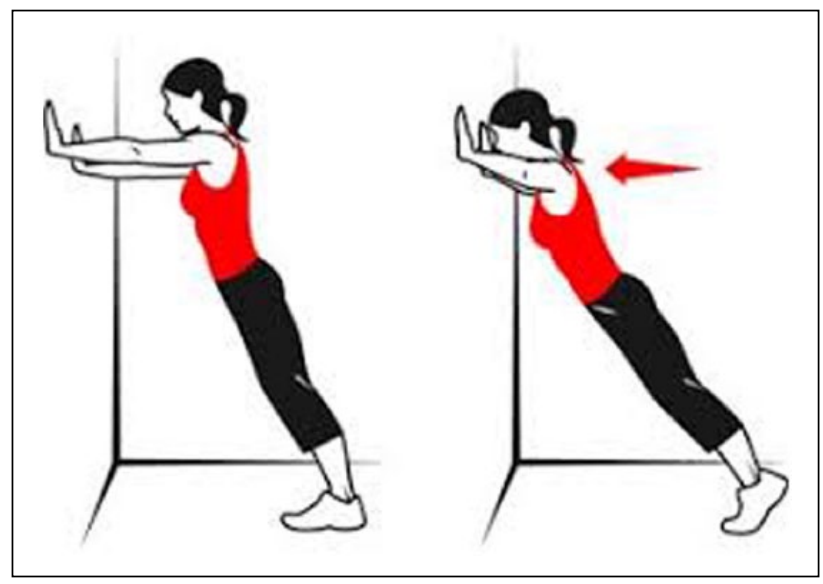

Figura 3.

Dichiarazione di assenza di conflitto di interessi

Gli Autori dichiarano di non avere conflitti di interessi. 


\section{Finanziamenti}

Gli Autori dichiarano di non aver ricevuto finanziamenti specifici da qualsiasi ente nei settori pubblico, privato o senza fini di lucro.

\section{Bibliografia}

1. Archer E and Blair SN. Physical Activity and the Prevention of Cardiovascular Disease: From Evolution to Epidemiology. Progress in Cardiovascular Diseases 2011; 53: 387-396.

2. Jeremy EN. Born to run? Journal of Experimental Biology 2005; 208. vi doi: $10.1242 /$ jeb.01468

3. White LA. Energy and the evolution of culture. Am Anthropologist 1943; 45:335-356.

4. Kavanagh T. Exercise in the primary prevention of coronary artery disease. Can J Cardiol 2001; 17:155-161.

5. Park RJ. Human energy expenditure from Australopithecus afarensis to the 4-minute mile: exemplars and case studies. Exerc Sport Sci Rev 1992; 20:185-220.

6. Ristinen RA and Kraushaar JP. Energy and the environment. Hoboken: John Wiley \& Sons, Ltd; 200.

7. Bortz WM II. The disuse syndrome. West J Med 1984; 141:691-694.

8. Raichlen DA, Pontzer H, Harris JA, et al. Physical activity patterns and biomarkers of cardiovascular disease risk in hunter-gatherers. Am J Hum Biol 2017; 29(2). doi: 10.1002/ ajhb.22919.

9. Caselli G, Mesle F and Vallin J. Epidemiologic transition theory exceptions. Genus 2002; 9:9-51.

10. Fetter B, Coelho PRP, Rogers J and Nelson MC. Forum: The Epidemiological Transition. Forum: The Health Transition Review 1997; 7 hdl:1885/40188

11. Blair SN, Kohl HW 3rd, Paffenbarger RS Jr, Clark DG, Cooper KH and Gibbons LW. Physical fitness and all-cause mortality. A prospective study of healthy men and women. JAMA 1989; 262(17):2395-2401.

12. Martin CK, Church TS, Thompson AM, et al. Exercise dose and quality of life: a randomized controlled trial. Arch Intern Med 2009; 169:269-278.

13. Booth FW and Chakravarthy MV. Cost and Consequences of Sedentary Living: New Battleground for an Old Enemy,
2002. President's Council of Physical Fitness and Sports Research Digest, Series 3, No. 16. President's Council on Physical Fitness and Sports, Washington, DC, 2002.

14. Thompson PD, Buchner D, Pina IL, et al. Exercise and physical activity in the prevention and treatment of atherosclerotic cardiovascular disease: a statement from the Council on Clinical Cardiology (Subcommittee on Exercise, Rehabilitation, and Prevention) and the Council on Nutrition, Physical Activity, and Metabolism (Subcommittee on Physical Activity). Circulation 2003; 107:3109-3116.

15. Biddle SJ and Asare M. Physical activity and mental health in children and adolescents: a review of reviews. Br J Sports Med 2011; 45(11):886-895. doi:10.1136/ bjsports-2011-090185

16. Szostak R. Interdisciplinary best practices for adapted physical activity. Quest 2016; 68(1):69-90. doi:10.1080/0 0336297.2015.1117001

17. Howley ET. Type of activity: resistance, aerobic and leisure versus occupational physical activity. Med Sci Sports Exerc 2001; 33(6 Suppl):S364,S369; discussion S419-20.

18. Hare DL, Ryan TM, Selig SE, Pellizzer AM, Wrigley TV and Krum H. Resistance exercise training increases muscle strength, endurance, and blood flow in patients with chronic heart failure. Am J Cardiol 1999; 83(12):1674-1677, A7.

19. Hurley BF and Roth SM. Strength training in the elderly: effects on risk factors for age-related diseases. Sports Med 2000; 30(4):249-268.

20. Frauman DC. The relationship between physical exercise, sexual activity, and desire for sexual activity. The Journal of Sex Research 1982; 18:1,41-46.

21. Wang CY, Haskell WL, Farrell SW, et al. Cardiorespiratory fitness levels among US adults 20-49 years of age: findings from the 1999-2004 National Health and Nutrition Examination Survey. Am J Epidemiol 2010; 171(4):426-435.

22. Begalle RL, DiStefano LJ, Blackburn T and Padua DA. Quadriceps and hamstrings coactivation during common therapeutic exercises. Journal of Athletic Training 2012; 47 : 396-405. 